\title{
Vaccine and immune cell therapy in non-small cell lung cancer
}

\author{
Helena Oliveres $^{1,2 \#}$, Christian Caglevic ${ }^{3 \#}$, Francesco Passiglia ${ }^{1,4}$, Simona Taverna ${ }^{1,5}$, Evelien Smits ${ }^{5,6}$, \\ Christian Rolfo ${ }^{7}$
}

${ }^{1}$ Phase I-Early Clinical Trials Unit, Antwerp University Hospital, Edegem, Belgium; ${ }^{2}$ Department of Oncology, Parc Taulí Hospital, Sabadell, Spain; ${ }^{3}$ Oncology Department, Clinica Alemana Santiago, Santiago, Chile; ${ }^{4}$ Department of Surgical, Oncological and Oral Sciences, Section of Medical Oncology Palermo, University of Palermo, Palermo, Italy; ${ }^{5}$ Center for Oncological Research Antwerp, University of Antwerp, Antwerp, Belgium; ${ }^{6}$ Center for Cell Therapy and Regenerative Medicine, Antwerp University Hospital, Edegem, Belgium; ${ }^{7}$ University of Maryland Marlene and Stewart Greenebaum Comprehensive Cancer Center, Baltimore, MD, USA

\#These authors contributed equally to this work.

Correspondence to: Prof. Dr. Christian Rolfo, MD, PhD, MBA. University of Maryland Marlene and Stewart Greenebaum Comprehensive Cancer Center, Baltimore, MD, USA. Email: christian.rolfo@umm.edu.

\begin{abstract}
Despite new advances in therapeutics, lung cancer remains the first cause of mortality among different types of malignancies. To improve survival, different strategies have been developed such as chemotherapy combinations, targeted therapies and more recently immunotherapy. Immunotherapy is based on the capability of the immune system to differentiate cancer cells from normal cells to fight against the tumor. The two main checkpoint inhibitors that have been widely studied in non-small cell lung cancer (NSCLC) are PD-1/PD-L1 and CTLA-4. However, interactions between tumor and immune system are much more complex with several different elements that take part and probably many new interactions to be discovered and studied for a better comprehension of those pathways. Vaccines are part of the prophylaxis and of the treatment for different infectious diseases. For that reason, they have allowed us to improve global survival worldwide. This same idea can be used for cancer treatment. First reports in clinical trials that used therapeutic vaccines in NSCLC were discouraging, but currently vaccines have a new chance in cancer therapy with the identification of new targetable antigens, adjuvant treatments and most interestingly, the combination of vaccines with anti-PD-1/PD-L1 and anti-CTLA-4 drugs. The aim of this article is to describe the scientific evidence that has been reported for the different types of vaccines and their mechanisms of action in the fight against NSCLC tumors to improve disease control.
\end{abstract}

Keywords: Cancer vaccines; immunotherapy; immune cell therapy; CAR T-cell therapy; lung cancer

Submitted Mar 16, 2018. Accepted for publication May 17, 2018.

doi: $10.21037 /$ jtd.2018.05.134

View this article at: http://dx.doi.org/10.21037/jtd.2018.05.134

\section{Introduction}

Lung cancer is the second most frequent malignancy worldwide after non-melanocytic skin cancer (1). Among the male population, during 2012, lung cancer was the second tumor in incidence and the first in mortality. In this same year, among the female population, lung cancer was the third malignancy in incidence after breast and colorectal cancer and the second in mortality (2). Considering all lung malignancies, the majority of the cases (almost $85 \%$ ) are classified as non-small cell lung cancer (NSCLC).
Depending on the stage and on the performance status (PS), different modalities of treatment may be used to treat lung cancer. For non-metastatic resectable NSCLC patients, the standard of care is surgery followed or not by adjuvant chemotherapy (3). For resectable stage III A and unresectable non-metastatic disease chemoradiation is a treatment option that addresses a curative intention too (4). In 2017, a phase III clinical trial showing the benefit of Durvalumab was published. This trial (Pacific trial) included patients with stage III NSCLC who did not have disease progression after two or more cycles of platinum- 
based chemoradiotherapy (5). The median progressionfree survival (PFS) was 16.8 months [95\% confidence interval (CI), 13.0-18.1] for the Durvalumab arm versus 5.6 months (95\% CI, 4.6-7.8) for placebo. Based on this data, Durvalumab, as adjuvant treatment, was the first immunotherapy drug to be approved after definitive chemoradiation in non-metastatic unresectable NSCLC patients.

For NSCLC advanced stage not amenable to be treated with curative intention, including non-metastatic unresectable and metastatic NSCLC patients, prognosis is poor, with a median overall survival (OS) lesser than 12 months. Only $3.5 \%$ of those patients will survive 5 years after diagnosis (6). Cisplatin-based chemotherapy demonstrated to increase the OS and quality of life when compared with best supportive care (BSC) (7) and it is still the standard of care in the first line setting unless targetable mutations or high PD-L1 expression are present.

Understanding and achieving more knowledge about the mechanisms, mutations and pathways that are involved in NSCLC, has taught us more about tumor heterogeneity, tumor burden and tumor microenvironment, but also, mostly during this last decade, it has provided us new tools for targetable mutations (8) and immunotherapeutic drugs development. Those developments have improved OS and also diminished toxicity when compared with the standard of care, cytotoxic chemotherapy, among NSCLC patients.

Tyrosine kinase inhibitors (TKIs) [epidermal growth factor receptor (EGFR) and anaplastic lymphoma kinase (ALK)] have notably improved median OS to over 30 months in selected patients with metastatic or unresectable NSCLC disease $(9,10)$. Currently, immunotherapy-based treatment has a great role in metastatic NSCLC. Pembrolizumab was approved in advanced disease as the first line of treatment in patients with $\geq 50 \%$ expression of PD-L1 and EGFRALK negative expression (11). Besides Pembrolizumab other immunotherapy drugs such as nivolumab (anti-PD-1) $(12,13)$ and atezolizumab (anti-PD-L1) (14) have been approved in metastatic NSCLC patients as a second line of treatment after progression to a cisplatin-doublet-based first line of treatment.

In contrast to the newly developed immune checkpoint inhibitors, vaccines have been an old weapon in medicine, mostly used for infectious disease prevention and to a lesser extent also developed in the cancer field. The concept of vaccines is to stimulate antigen-specific immunity response by presenting a pathogen or tumor-associated antigens (TAAs). This idea was initially developed in 1796 by
Edward Jenner and who had an innovative and important contribution to eradicate smallpox (15). Later, in 1891, William Coley introduced the idea of treating malignancies stimulating immunity (16). He observed spontaneous tumor regressions in patients after infectious diseases such as erysipelas. Coley injected lived Streptococcus Pyogenes inside patients' tumors with the idea that the immune system would eradicate the infection, and as a collateral effect, the tumor could also be destroyed.

Vaccines in cancer have been used both for prevention and for treatment as well. Some relevant examples are the recombinant human papillomavirus (HPV) (17) and the Bacillus Calmette-Guérin (BCG) vaccines. For NSCLC, the CIMAvax Epidermal Growth Factor vaccine has been approved in Cuba, Peru and Venezuela for stage IIIb and IV after progression of a first line chemotherapy (18) and Racotumomab in Argentina and Cuba as maintenance therapy for advanced NSCLC after a primary treatment. Recently, the genetically engineered immune collected cells from patients, chimeric antigen receptors (CAR) modified $\mathrm{T}$ cells, was approved by FDA for the treatment of some hematological malignancies, becoming a new therapeutic tool using immune-system pathways looking to treat different types of cancer.

Furthermore, different studies have been set up to investigate the efficacy of vaccines in early stages as an adjuvant therapy and in advanced stages as a palliative treatment. In this review we will discuss the current evidence of vaccines and CAR T cell use in NSCLC.

\section{The immune system against the tumor}

To date, several important players in the antitumor immune response have been identified, and together with a deeper knowledge of the immune system mechanisms has helped us to understand better how it works and how it fights against tumor cells. The activation of cytotoxic $\mathrm{T}$ lymphocytes (CTL) is carried out in several phases. Dendritic cells (DCs) as professional antigen presenting cells (APC) and macrophages migrate from the tumor to the lymph node with the antigen on their surface and present the tumor antigen to the CD4+ $\mathrm{T}$ helper cells (Th) by the union between an MHC-II molecule + epitope and a specific T cell receptor on the CD4 $\mathrm{T}$ cell. If also co-stimulation is present, such as CD40 (on the APC)-CD40L (on the CD4 $\mathrm{T}$ cell), this binding activates the APC to present MHCI-binding epitopes to CTL. CTL get activated if also a costimulatory signal is provided, e.g., through binding of 


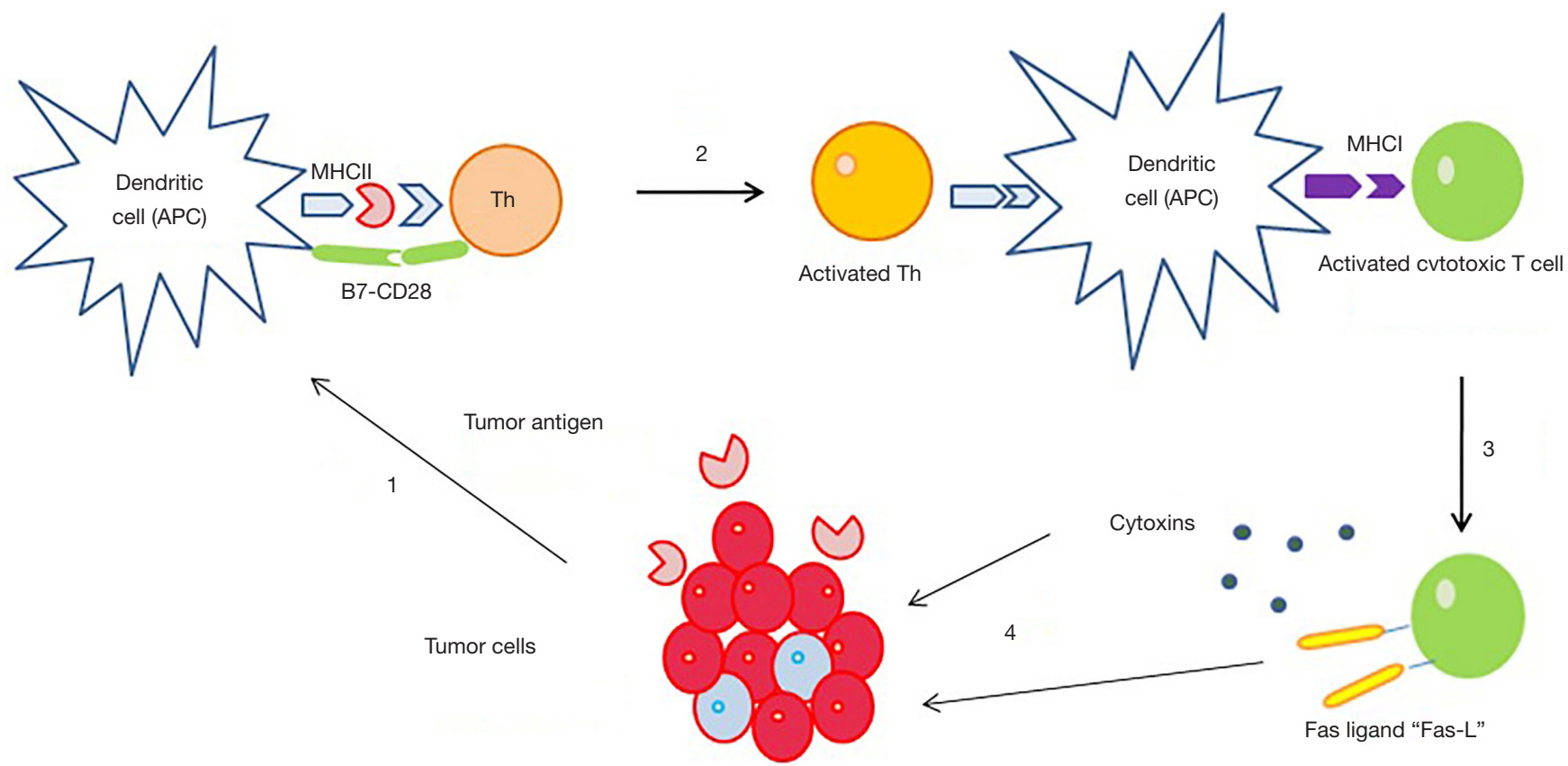

Figure 1 Tumor antigens recognized by T cells. APC, antigen presenting cell; MHC, major histocompatibility complex.

B7 (on the APC) with CD28 (on the CTL). The activated CTL travels from the lymph node to the tumor to attack the tumor cell. In the tumor, the CTL recognize the tumor antigens of the tumor cells and destroy them by two main mechanisms. These mechanisms include: (I) releasing granules in the contact zone that contain perforin (induces pore formation in the tumor membrane) and granzymes (induces apoptosis); and (II) the expression of FAS-L that induces apoptosis in tumor cells that express FAS (19) (Figure 1).

B lymphocytes activate the humoral immune response. $\mathrm{CD} 4+\mathrm{T}$ helper cells stimulate the maturation of $\mathrm{B}$ cells into plasma cells and lead to the production of antigen-specific antibodies (20).

Tumor cells can have different strategies to escape from the immune attack. Immune responses impose pressure on tumor cells, resulting in selection of tumor cells that can escape. Highly proliferating tumors can lose expression of tumor antigens or MHC molecules by mutations or deletions of genes. Tumor antigens can also be masked from immune recognition by glycocalyx molecules.

They prevent T-cell CD4+ mediated responses by repressing genes encoding the B7-1 and B7-2 costimulators. The tumor may also release interleukins such as IL-10 or TGF- $\beta$ to inhibit the immune response.

These different ways of eluding the immune system explain why creating an effective vaccine is a complex process. To improve the effectiveness of vaccines various strategies have been developed. For example, a strategy is to administer the vaccine together with a strong adjuvant that helps to increase the host's immune response. To assess the efficacy of combining different cancer treatment with vaccines several clinical trials are ongoing.

\section{Types of therapeutic cancer vaccines in NSCLC}

The objective of vaccination therapy is to provoke an adaptive antitumor immune response (21). Vaccines should generate a potent $\mathrm{CD} 4+$ and $\mathrm{CD} 8+\mathrm{T}$ cell response and also should be tumor specific, avoiding that normal host cells were targeted (22).

During the last decade, vaccines for NSCLC have been studied in several large phase III trials. They showed a good toxicity profile and tolerability, however, they were not able to demonstrate survival benefits despite promising results that were achieved in preliminary phase II randomized trials.

Vaccines are classified according to the type of targeted antigen (Table 1) (23).

Different vaccines have been developed to stimulate the immune system against a broad range of TAAs (24). There are 4 main types of lung tumor antigens (25). The family of gangliosides (GM2, GD2, GD3, Fuc-GM1) is a broad family of glycosphingolipids found on the outer 
Table 1 Types of vaccines investigated in NSCLC

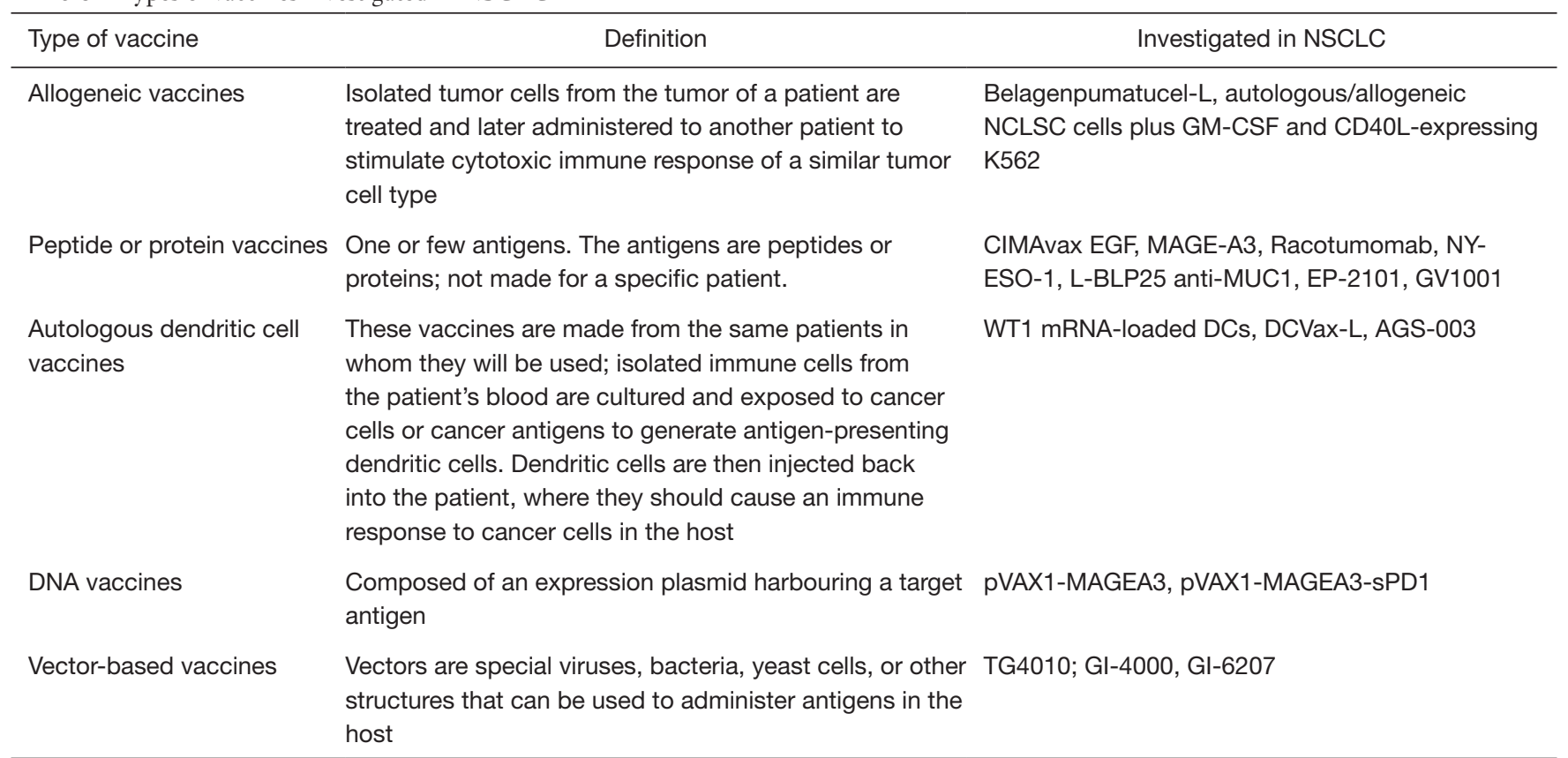

NSCLC, non-small lung cancer cell; GM-CSF, granulocyte stimulating factor; EGF, epidermal growth factor; MAG, melanoma-associated antigen; NY-ESO-1, New York esophageal squamous cell cancer; L-BLP25, BLP25 liposome vaccine; MUC1, Mucin 1; DCs, dendritic cells.

cell membrane that are involved in cell communication, regulation of the immune response, and cancer progression $(26,27)$. They are overexpressed in tissues of neuroectodermal origin, and particularly in tumors such as melanoma, neuroblastoma, sarcoma and lung cancers (28). The second type of TAAs is produced by a unique mutation of a normal gene such as K-ras, EGFR and $\mathrm{p} 53$. The third type is the cancer testis antigens (CTA) and includes New York esophageal squamous cell cancer (NY-ESO-1), melanoma-associated antigen (MAG), GAGE and BAGE. Their normal expression is restricted to male germ cells in the testis and in some cases also in ovary and trophoblast. In some malignancies gene regulation is disrupted, resulting in CTA expression in a proportion of different tumors types (29). CTA are immunogenic proteins that are related with poor prognosis in NSCLC. The fourth type is antigens expressed by normal cells that are expressed in higher levels in cancer cells. These antigens include Mucin 1 (MUC1), WT-1, carcinoembryonic antigen (CEA), survivin and Her2/neu.

\section{Allogeneic vaccines}

In allogeneic vaccines, the antigen source is non-self-cancer cells. They are produced from cancer cells of another patient with the same tumor type, then they are modified and processed prior to their use (30).

\section{Belagenpumatucel-L}

Belagenpumatucel-L is prepared by transfecting 4 radiated allogeneic NSCLC cell lines (H460, RH2, SKLU-1, H520), including 2 adenocarcinomas, one squamous and one large cell carcinoma, with a plasmid containing transforming growth factor $\beta 2$ (TGF- $\beta 2$ ) antisense transgene (31). TGF- $\beta$ is a negative regulator of CTL. Perturbation of TGF- $\beta$ signaling is linked to tumor progression (32), high levels lead to immunosuppression and are related with worse prognosis in NSCLC patients. In this vaccine, the antisense transgene inhibits TGF- $\beta 2$ with the intention to increase immunogenicity.

Two phase II clinical trials demonstrated the safety and efficacy of Belagenpumatucel-L in patients with NSCLC. The first one, which was published on 2006, included a total of 75 stage II, IIIA, IIIB and IV patients (33). The second study was published on 2009 included 21 advanced NSCLC patients (34). Both trials, besides showing a consistent result on safety and response, also suggested an inverse correlation between circulating tumor cell levels and survival.

A phase III trial that studied this vaccine as maintenance 
therapy was published on 2015, its primary endpoint was OS. A total of 532 stage III/IV NSCLC patients that had no disease progression after a first line of platinum-based chemotherapy were enrolled. Patients were randomized 1:1 to receive maintenance therapy with Belagenpumatucel-L or placebo. The primary endpoint was not reached, no difference in OS was met (20.3 months in the treatment arm vs. 17.8 months in the placebo arm, HR 0.94, $\mathrm{P}=0.594$ ). No difference in PFS was reached either between both arms (4.3 versus 4 months in treatment arm and placebo group respectively, HR 0.99, $\mathrm{P}=0.947$ ). Despite the negative results in OS and PFS, a prespecified Cox regression analysis showed a significant benefit in survival among patients that started vaccination within 12 weeks of the completion of chemotherapy $(\mathrm{P}=0.002)$ and among patients that underwent prior radiotherapy treatment $(\mathrm{P}=0.032)$ (35).

Although not in a vaccination context, also other TGF- $\beta$ inhibitors are being studied in NSCLC with similar mechanisms of action. Galunisertib is a small molecule inhibitor developed by Eli Lilly. At 2016 ASCO meeting, a phase II randomized clinical trial focusing on unresectable stage II-IV pancreatic cancer patients, assessed the combination of Galunisertib plus Gemcitabine combination versus Gemcitabine alone, showing benefit in OS and PFS (36). Currently, a phase Ib/II study of Galunisertib in combination with Nivolumab in refractory solid tumors including NSCLC patients is ongoing.

\section{Autologous or allogeneic NSCLC cells plus GM.CD40L expressing K562 cells}

A phase I trial tested the vaccine, irradiated autologous tumor cells plus GM.CD40L bystander cells, in patients with stage IV NSCLC. Twenty-one patients received 3 intradermal vaccine injections every 28 days. No toxicity treatment related was reported. Reported results showed that patients had stable disease and some of them developed tumor specific T-cell responses (37).

A phase II study was conducted for refractory advanced lung adenocarcinoma patients. 24 heavily pretreated patients were recruited with a median of 4 previous lines of systematic therapy. The vaccine (GM.CD40L plus tumor cell-based vaccine) was administered intradermally in the axillary and inguinal lymph node basins every 2 weeks for the first 4 weeks and then monthly for the next 3 months. Cyclophosphamide pretreatment was included and all-transretinoic acid (ATRA) was added to induce differentiation of immature DCs at the local vaccine site. The primary endpoint, inducing radiologic tumor regression, was not reached. Median OS was 7.9 months and median PFS was only 1.7 months (38).

With the aim to improve the effectiveness of the vaccine, adjuvant drugs have been tested. One example is $\mathrm{C}-\mathrm{C}$ motif chemokine ligand 21 (CCL21). Chemokines play essential roles in tumor biology: leukocyte recruitment, tumor cell growth, angiogenesis and metastasis. When $\mathrm{T}$ cells are exposed to both CCL21 and DCs, there is an increase in the immune response. A phase 1-2 randomized trial of a vaccine consisting of the GM.CD40L bystander cells and an equivalent number of allogeneic tumor cells with or without CCL21 was assessed in adenocarcinoma lung patients that failed to a first line of chemotherapy. The combination was well tolerated however expected outcomes such as OS and PFS were not improved when adding CCL21 to the vaccine (39).

\section{Antigens peptide or protein vaccines}

\section{CIMAvax epidermal growth factor vaccine}

EGFR overexpression is associated with uncontrolled proliferation, angiogenesis, anti-apoptotic signals, metastasis and invasiveness. EGFR is widely expressed in many cell types including epithelial and mesenchymal cells. EGFR is expressed in squamous malignancies of the head and neck, colorectal, breast, prostate, bladder, ovary and lung cancer (40). EGFR gene mutations were the first mutations to be targeted with positive outcomes for the treatment of NSCLC.

Among NSCLC patients the incidence of EGFR mutations is around $27 \%$ being more common in patients with adenocarcinoma histology, in never or light smokers patients, in women and in the Asiatic population. If properly treated it is associated with better prognosis when compared with EGFR wild type (41). The main activating EGFR mutations have been identified in exons 18 to 21 of the tyrosine kinase domain, representing from $85-90 \%$ of all the EGFR mutations (42). Available drugs that target EGFR are: small molecule EGFR TKIs gefitinib and erlotinib (first generation non-covalent inhibitors), afatinib (second-generation covalent inhibitor), Osimertinib (third generation TKI, targeting EGFR-T790M resistance mutation) and monoclonal anti-EGFR antibody Cetuximab.

The CIMAvax-EGF vaccine is a chemical conjugate of EGF with the P64 protein derived from the Meningitis B bacteria and Montanide ISA 51 as adjuvant (43). As earlier mentioned this vaccine was developed in Cuba and its use is approved in Cuba, Venezuela and Peru for the treatment of 
stage IIIB and IV NSCLC that have progressed after a first line of chemotherapy.

CIMAvax-EGF induces immune responses against EGF, a molecular driver of cancer cell, with the purpose to block cancer cell proliferation. CIMAvax-EGF demonstrated to be safe and immunogenic in advanced NSCLC patients in a phase II randomized controlled study (44). This study included 80 stage IIIB/IV NSCLC patients after they completed a first-line chemotherapy. They were randomized to BSC or to vaccination treatment. Patients were assessed for anti-EGF antibody response. Good antibody responders (GAR) were defined as those that reached an anti-EGF antibody titer equal or higher than 1:4,000 serum dilution. Patients that reached lower antibody titers were classified as poor antibody responders (PAR). Good anti-EGF antibody response was obtained in $51.3 \%$ of vaccinated patients. GAR patients survived significantly more (median survival of 11.7 months) than those with PAR (median survival of 3.6 months). Adverse events were observed in less than $25 \%$ of cases and were classified as grade 1 and 2 .

In August 2016, a phase III study was published to show the results of OS, safety, immunogenicity, and EGF concentration in serum after CIMAvax-EGF vaccine (45). After completing a first line of chemotherapy treatment, 405 stage IIIB/IV NSCLC patients were randomly assigned $(2: 1)$ to the vaccine plus BSC arm or to a control group (BSC). Before the first vaccine administration a low dose of cyclophosphamide was given. Each vaccine dose was administered every 2 weeks for 4 doses (induction period) and since then monthly.

In the vaccine group, $81 \%$ of the patients received 4 vaccines doses. The survival benefit was significantly better (HR, 0.77; $\mathrm{P}=0.036)$ in the treatment arm. Median survival was 12.4 months for the vaccine arm versus 9.4 months for the control arm. Moreover, median survival was higher (14.6 months) for vaccinated patients with high EGF concentration at baseline.

High EGF concentration at baseline was a good predictive biomarker of the vaccine activity and also a poor prognostic biomarker among the non-treated population. The proportion of CD8+CD28- cells, CD4 cells, and the CD4/CD8 ratio after first-line of chemotherapy was also associated with CIMAvax-EGF clinical benefit.

In January 2017, a randomized phase I/II clinical trial with CIMAvax-EGF in combination with nivolumab (NCT02955290) has started to assess results in second line for advanced NSCLC at Roswell Park Cancer Institute in Buffalo, New York, in collaboration with the Center of
Molecular Immunology in Cuba.

\section{MAGE-A3}

CTA are immunogenic and highly specific for cancer. MAGE was the first CTA to be discovered (46). MAGE expression is found in $30-50 \%$ of NSCLC samples, being more frequent in squamous cell lung cancer histology (47).

The safety of MAGE-A3 as adjuvant therapy in patients with resected MAGE-A3 positive NSCLC was studied in the phase III MAGRIT trial. A total of 2,312 resected stage IB, IIA and IIIA NSCLC patients, who had been treated or not with adjuvant chemotherapy, were randomized 2:1 to receive the vaccine plus the adjuvant AS15 or to placebo. The primary endpoint was disease-free survival (DFS). Final results did not show a benefit in DFS in the MAGE-A3 immunotherapeutic group when compared with the placebo group (60.5 vs. 57.9 months respectively) (48).

Another phase I/II dose escalation trial testing Adenovirus Vaccine Expressing MAGE-A3 and MG1MAGEA3 in combination with Pembrolizumab, is currently recruiting NSCLC patients who have radiological progression after at least 1 cycle platinum-doublet chemotherapy (NCT02879760).

\section{NY-ESO-1}

NY-ESO-1 is another CTA candidate for specific immune recognition of cancer due to its frequent occurrence in cancer samples and its restricted expression in somatic tissues (49). NY-ESO-1 is approximately expressed in $30 \%$ of lung cancer specimens (50).

The relation between the expression of NY-ESO-1 and therapy response has already been assessed. John et al studied a group of patients with pathological N2 NSCLC tumors that underwent surgery, neoadjuvant and adjuvant chemotherapy. Results supported NY-ESO-1 as a useful prognostic factor and as a predictive factor for significant benefit of the adjuvant and neoadjuvant chemotherapy treatment (51).

In 2005, Murphy et al. used a recombinant NYESO-1 protein produced in Escherichia Coli (52) and demonstrated induction of specific antibodies in serum, as well as activation of CD4+ and CD8+ T cells. Because it is a promising target antigen, there are several clinical trials ongoing as monotherapy or as combination treatment that are assessing these findings as an area to explore in cancer therapy. A phase I multicenter trial is recruiting patients to evaluate the safety and the immune response of ID-LV305 (immunotherapy agent that targets DCs) in patients with 
advanced cancer whose tumors express the NY-ESO-1 protein (NCT02122861).

IDC-G305 is a new vaccine consisting of recombinant NY-ESO-1 antigen and the adjuvant GLA-SE. It is under study in solid malignancies to evaluate safety and immune response (NCT02015416). GLA-SE is a potent vaccine adjuvant consisting of the synthetic toll-like receptor 4 (TLR4) agonist Glucopyranosyl Lipid Adjuvant (GLA) formulated in a squalene-in-water emulsion (SE) (53).

\section{Racotumomab (formerly known as IE10)}

Racotumomab is an anti-idiotype murine IgG1 directed to NeuGcGM3 ganglioside, a tumor antigen expressed in NSCLC tumors.

Neninger et al. conducted a phase I clinical trial in patients with small cell lung cancer (SCLC) that had partial or complete response after chemotherapy and/or radiotherapy (6 patients with limited stage-disease and 3 patients with extensive-stage disease). Patients received 4 biweekly injections with $2 \mathrm{mg}$ Racotumomab-alum, followed by six more doses every 28 days. No evidence of severe adverse effects and a prolonged survival was reported (54).

A compassionate use basis study administrated the vaccine in 34 stage III b and 37 stage IV NSCLC patients after they received standard chemotherapy and radiotherapy. Patients received 5 biweekly injections, followed by 10 doses at 28-day intervals. Patients who entered the study with partial response or stable disease and with a PS of $0-1$ after the first line of chemo/radiotherapy had a median survival of 11.5 months, however, patients that achieved disease progression after chemo/radiotherapy or that started vaccination with PS 2 had a median survival of only 6.5 months (55). In 2014, a randomized, multicenter, placebo-controlled phase II/III trial for advanced NSCLC patients who achieved stable disease after a first line of chemotherapy was published. Median OS was 8.2 in the vaccine group and 6.8 months in the placebo group (HR, 0.63; 95\% CI, 0.46-0.87; $\mathrm{P}=0.004$ ). Median PFS in vaccinated patients was 5.3 versus 3.9 months for placebo (HR, 0.73; 95\% CI, 0.53-0.99; $\mathrm{P}=0.039$ ). No severe adverse effects were reported (56). Based on these results, Racotumomab was launched in 2013 in Cuba and Argentina as an intradermal injection for the treatment of patients with advanced stage NSCLC.

The impact of using Racotumomab-alum in patients with advanced NSCLC who have achieved an objective response (partial or complete response) or stable disease after standard first-line treatment is currently being explored in a randomized, multicenter, open label phase III study. The primary end point of this trial and secondary end points include PFS, safety and tolerability, final results have not been reported yet (NCT01460472).

\section{BLP25 liposome vaccine (anti-MUC1)}

Cell surface associated MUC1 is a member of the mucin family. Mucins are $\mathrm{O}$-glycosylated proteins that have a role in forming protective mucous barriers on epithelial surfaces and participate in intracellular signaling. Over expression, aberrant intracellular localization and changes in glycosylation of this protein have been associated with carcinomas. MUC1 is overexpressed and aberrantly glycosylated in NSCLC samples (57).

The BLP25 liposome vaccine (L-BLP25) targets MUC1 and induces a cellular immune response that may lead to immune rejection of tumor tissues that express MUC1 antigen (58).

In 2005, a phase IIB trial was published of BLP-25 vaccine as maintenance therapy for stage IIB-IV NSCLC patients that had achieved stable disease or objective response after a first line chemotherapy. Patients were randomized in two arms, L-BLP25 plus BSC or BSC alone (59). Despite a 4 months absolute benefit in the treatment arm there was not statistical difference in OS between both arms (17.4 months in the vaccine arm versus 13 months for BSC arm; HR, 0.739; 95\% CI, 0.509-1.073; $\mathrm{P}=0.112$ ). A published update in 2011 reported that median OS remained statistically non-significant. However, a significant increase in 3-year survival rate was observed (31\% for vaccine arm, 17\% for BSC arm, $\mathrm{P}=0.035$ ) (60).

The phase III START trial was published in Lancet Oncology in 2014 (61). This was an international, doubleblind trial that included patients with unresectable stage III NSCLC who had completed chemoradiotherapy and had confirmation of stable disease or objective response. Patients received L-BLP25 or placebo in a 2:1 randomization. There was no difference in OS. Median OS was 25.6 months (95\% CI, 22.5-29.2) in the treatment arm vs. 22.3 months (19.6-25.5) in the placebo arm (adjusted HR, 0.88; 95\% CI, $0.75-1.03 ; \mathrm{P}=0.123)$.

Further studies were initiated such as the phase III START2 (62) and INSPIRE (63) trials for the treatment of unresectable, locally advanced stage III NSCLC. However, the sponsor decided to discontinue the current clinical program due to the results of the EMR 63325009 trial. This was a randomized, double-blind, placebocontrolled phase I/II study in Japanese patients with 
stage III unresectable, locally advanced NSCLC who had received concurrent or sequential chemoradiotherapy, with a minimum of two cycles of platinum-based chemotherapy and a radiation dose $\geq 50$ Gy (64). The results of this study showed no benefit in OS (primary endpoint) neither for any of the secondary endpoints (PFS, time to progression and time to treatment failure). The biopharmaceutical company division made the recommendation to stop the investigational treatment for patients in the EMR 63325009 study in Japan and consequently all other clinical trials with L-BLP25 (Tecemotide) in NSCLC patients worldwide.

\section{Autologous DCs vaccines}

DCs are APC of the innate immune system that form a bridge between innate and adaptive immunity. They control different areas of the immunity such as the stimulation of naive $\mathrm{T}$ lymphocytes, induction of primary antibodies responses and the maintenance of tolerance against selfantigens (65). DCs have all the elements to properly initiate an antigen specific immune response. Therefore they are attractive to be used as cancer vaccine and different trials have already shown biological activity (66).

Currently there are several trials, some of them already completed or no longer recruiting, that are addressing the potential benefits of autologous DC vaccines in different stages of NSCLC and SCLC, but also they are exploring their utility in some other malignancies as well.

\section{DNA vaccines}

DNA vaccines are based on the direct introduction of a plasmid containing the DNA sequence encoding the antigen against which an immune response is sought, aiming for trough the in-situ production of the target antigen.

pVAX1-MAGEA3-sPD1 is a DNA vaccine constructed by cloning the Melanoma Associated Antigen 3 (MAGE-A3) gene and murine sPD1 genes linked by Furin-2A (F/2A) into pVAX1. The vaccine was tested in mice and the results showed enhanced $\mathrm{B}$ and $\mathrm{T}$ cell responses and potent inhibition of tumor growth.

A published phase I trial reported the results ion safety and efficacy of the Elenagen vaccine, a plasmid that encodes p62 (SQSTM1). This vaccine has 2 main mechanisms of action: inducing an immune response against p62 (protein in epithelial cells that induce oncogenic transformation) and controlling of systemic chronic inflammation. The trial included 27 patients with advanced solid tumors were recruited, including 2 lung cancer patients. No serious adverse effects were reported. Most of the patients achieved stable disease for at least 8 weeks (67).

\section{Vector vaccines}

\section{TG4010 (MVA-MUC1-IL-2)}

TG4010 is a therapeutic cancer vaccine based on a viral vector, a Modified Vaccinia Virus Ankara (MVA), that encodes for human MUC1 and for interleukin 2 as well (68).

A good safety profile and early signs of clinical and biological activity were observed in a phase I clinical trial conducted by Rochlitz et al. in 2003 (69). Thirteen patients with different solid tumors including lung cancer were treated by repeated intramuscular injections with increasing doses of TG4010. Four of the 13 patients achieved stable disease. One lung cancer patient showed a significant decrease in the size of its metastasis that lasted for 14 months.

A phase II randomized clinical trial that included 65 stage III B and IV NSCLC patients assessed the combination of TG4010 with the first line chemotherapy (cisplatin plus vinorelbine doublet) or the use of the vaccine without chemotherapy. According to RECIST criteria $29.5 \%$ of the patients that were treated in the combination arm achieved radiological response. The median time to progression was 4.8 months in the combination arm and the median OS was 12.7 months for the combination arm and 14.9 months in the vaccine alone arm. One-year survival was $53 \%$ and $60 \%$ for the combination arm and for the vaccine arm respectively. These results raised the hypothesis that TG4010 plus chemotherapy in the first line advanced or metastatic setting could improve the outcomes of chemotherapy alone (70).

A phase IIb clinical trial, assessing the combination of the vaccine and chemotherapy (cisplatin-gemcitabine doublet) in advanced NSCLC patients expressing MUC1 by immunohistochemistry (71) was published in 2008 . There were 2 arms, 74 patients were allocated to the combination therapy and 74 patients were allocated to the control group which received chemotherapy alone. Reported results showed 6 months PFS of $43.2 \%$ (32 of 74 patients; $95 \%$ CI, 33.4-53.5) in the TG4010 plus chemotherapy group, and $35.1 \%$ (26 of 74 patients; $95 \%$ CI, 25.9-45.3) in the control arm. Based on these results a phase IIb/III trial was conducted. The trial recruited 222 previously untreated patients with stage IV NSCLC cancer, negative EGFR mutation and with a MUC1 
Table 2 Ongoing clinical trials testing CAR T-cell therapy for NSCLC

\begin{tabular}{|c|c|c|c|c|}
\hline Clinical trial & Phase & Population & Target & Status \\
\hline NCT01869166 & I & EGFR+, refractory & EGFR & Recruiting \\
\hline NCT02414269 & I & Metastatic to the pleura & Mesothelin & Recruiting \\
\hline NCT02587689 & $\mathrm{I} / \mathrm{II}$ & Refractory, recurrent & MUC1 & Recruiting \\
\hline NCT03060343 & I & Refractory, Recurrent Metastatic & $\begin{array}{l}\text { Zeushield cytotoxic T } \\
\text { lymphocytes (Z-CTIs) }\end{array}$ & Recruiting \\
\hline NCT03029273 & $\mathrm{I} / \mathrm{II}$ & $\begin{array}{l}\text { Second line, Stage IIIb-IV, NY-ESO- } 1 \text { positive cells } \\
>10 \% \text { by IHC }\end{array}$ & NY-ESO-1 & Recruiting \\
\hline
\end{tabular}

CAR, chimeric antigen receptor; NSCLC, non-small lung cancer cell; EGF, epidermal growth factor receptor; NY-ESO-1, New York esophageal squamous cell cancer; L-BLP25, BLP25 liposome vaccine.

expression of at least $50 \%$ in tumor cells. Primary end point was PFS. The use of the triple-positive activated lymphocytes (TrPAL) as a biomarker was also investigated (72). Median PFS was 5.9 months (95\% CI, 5.4-6.7) in the TG4010 plus chemotherapy group and 5.1 months (4.2-5.9) in the chemotherapy alone group [HR, 0.74 (95\% CI, 0.55-0.98); one-sided $\mathrm{P}=0.019$ ]. These results also support the clinical value of the TrPAL as a biomarker.

Currently a phase II trial based on the combination between the vaccine and immunotherapy is ongoing in NSCLC. The rationale is that the TG4010 and Nivolumab combination may improve the effectiveness of the immune response to kill tumor cells (NCT00793208). Another phase 2 trial non-randomized trial has recently started to recruit patients to assess safety and feasibility of the combination of the vaccine, Nivolumab and chemotherapy in treatment naïve patients with advanced non-squamous NSCLC and $<50 \%$ of tumor cells expressing PD-L1 (NCT03353675).

\section{Adoptive T cell immunotherapy}

"Adoptive cell therapy" is another strategy in immunotherapy. Tumor-reactive lymphocytes from the patient are grown "ex vivo" and are then readministered to the patient as therapy. The most commonly used type of immune cells are CTL. Different types of T cells can be used for adoptive cell transfer: tumor-infiltrating lymphocytes (TILs), T-cell receptor (TCR)-modified T cells and CAR-modified T cells.

CAR T-cell therapy was the first cancer therapy using genetically engineered immune cells collected from patients, which was approved by the Food and Drug Administration (FDA). There are two CAR T-cell therapies that are approved: Tisagenlecleucel $\left(\mathrm{Kymriah}^{\mathrm{TM}}\right)$ for children and adults with advanced leukemia and Axicabtageneciloleucel (Yescarta $^{\mathrm{TM}}$ ) for patients with large-B-cell lymphomas whose cancer has progressed after receiving at least 2 prior treatment regimens.

Studies for NSCLC patients are limited, and the identification of a target is challenging. Current trials that are focused on this population are summarized in Table 2.

Robbins et al. evaluated the ability of adoptively transferred autologous $\mathrm{T}$ cells transduced with a TCR directed against NY-ESO-1 to mediate tumor regression in patients with metastatic melanoma and synovial cell sarcoma. Reported results showed that $61 \%$ of the synovial cell sarcoma and $55 \%$ of the melanoma patients achieved responses (73). Another report of a phase I/II clinical trial for multiple myeloma patients showed a PFS of 19.1 months for the group that received high affinity antiNY-ESO-1 and LAGE-1 specific TCR-T treatment (74). There is a trial ongoing for second line NSCLC aiming to assess the safety and tolerability of TAEST16001 (TAEST: TCR Affinity Enhancing Specific T cell Therapy, autologous $\mathrm{T}$ cells transduced with affinity enhanced NYESO-1 TCR) cell therapy (NCT03029273).

Not yet initiated, a phase I trial is designed to evaluate the effect of CAR T-cell in advanced lung cancer for patients where standard treatments failed. Investigators hypothesize that the CAR T cells targeting PD-L1 would improve the current efficacy of CAR-T cells. They 
designed and cloned a PD-L1 CAR gene that targets the PD-L1 expressed on tumor cells. PD-L1 CAR-T cells might effectively kill not only PD-L1 positive tumor cells in vivo, but also immunosuppressive cells inside tumors (NCT03330834).

Recently preliminary results were published of the specific CAR targeted to EphA2 (Hepatocellular Carcinoma A2). EphA2 is overexpressed in more than $90 \%$ of NSCLC but not significantly in normal lung tissue (75). The study concluded that $\mathrm{T}$ cells redirected to EphA2 by an EphA2specifc CAR have potent antitumor activity against NSCLC in vitro and in vivo, and could become a novel therapeutic agent for patients with NSCLC.

\section{Conclusions}

Lung cancer is the main cause of death due to malignancies worldwide. Most of the patients are diagnosed in advanced stages and will be not able to achieve a cure for their disease. Even more, many patients that are diagnosed in earlier stages will relapse after a curative intention of treatment that may include surgery, chemotherapy and radiation therapy.

Since the 80s until know, chemotherapy has been the cornerstone of treatment of metastatic disease with the purpose to improve survival and quality of life. Last decade targeted therapies have significantly improved survival among patients with metastatic disease that are carriers of specific mutations such as EGFR, ALK, and ROS1. Immunotherapy based on immune checkpoint inhibitors, mainly blocking PD-1/PD-L1 or CTLA4, has revolutionized the treatment in metastatic lung cancer with less toxicity compared with chemotherapy. Some patients that have impressive prolonged survival have allowed developing this powerful therapeutic weapon, probably opening a window for a cure of cancer.

Despite the enormous advances in NSCLC treatment in the recent past years, months and days, NSCLC patients still need more options for treatment. Since a long time, vaccines have been a powerful tool to prevent infectious disease allowing to decrease dramatically the risk to die for many contagious infections. Researchers have attempted by decades to create vaccines to fight against several types of cancer, unfortunately most of these have failed to prove a benefit in patients with solid tumors. Due to a better understanding of the tumor biology the immune system, immune phenotypes of patients and the tumor microenvironment, in addition to new technologies and pharmacological development, the gate has reopened to think of vaccines as a therapeutic option to increase results of current standard of care treatments in NSCLC. Most of the new vaccines have demonstrated to be safe and well tolerated in humans, but with low benefit in response rate, OS and PFS. Some trials have shown efficacy in OS and PFS such as Racotumomab when used as extended treatment after standard treatment for stage III B and IV NSCLC patients, however only a very small number of Latin American countries have given a formal approval for this vaccine to be used. Unfortunately, some vaccines that initially seemed to be promising; unfortunately failed to show survival benefit and resulted in several halted vaccination developing programs. Predictive and response biomarkers will probably help to select a better target NSCLC population that will benefit from specific vaccines.

Several trials are currently ongoing using vaccines in the adjuvant and metastatic setting. Among NSCLC patients, new combinations are being explored such as vaccines plus chemotherapy, vaccines plus immune checkpoint inhibitors, vaccines plus chemotherapy and immune checkpoint inhibitors. Research is also ongoing to find biomarkers for a better selection of patients. This information probably will provide us with the knowledge to explore vaccines addressed to NSCLC patients as a new alternative to improve outcomes in survival and quality of life for this population.

\section{Acknowledgements}

None.

\section{Footnote}

Conflicts of Interest: Dr. C Caglevic disclosures the following conflicts of interest: Speaker: MSD, GSK, Bayer, BMS, Boehringer Ingelheim, Tecnofarma, Roche; Principal Investigator: MSD, GSK, Bayer, Boehringer Ingelheim, Astellas, Roche, Astra Zeneca, BMS, Novartis; Advisory and Consulting: MSD, BMS, Bayer, Astra Zeneca, Boehringer Ingelheim, Lilly, Tecnofarma; Sponsored Educational Program: Boehringer Ingelheim, Tecnofarma, BMS, MSD. Dr. C Rolfo disclosures the following conflicts of interest: Speaker bureau: MSD, Novartis, Astra Zeneca, Guardant Health; Research: OncoDNA. The other authors have no conflicts of interest to declare.

\section{References}

1. Novello S, Barlesi F, Califano R, et al. Metastatic non- 
small-cell lung cancer: ESMO Clinical Practice Guidelines for diagnosis, treatment and follow-up. Ann Oncol 2016;27:v1-27.

2. Ferlay J, Soerjomataram I, Dikshit R, et al. Cancer incidence and mortality worldwide: sources, methods and major patterns in GLOBOCAN 2012. Int J Cancer 2015;136:E359-86.

3. Douillard JY, Rosell R, De Lena M, et al. Adjuvant vinorelbine plus cisplatin versus observation in patients with completely resected stage IB-IIIA non-small-cell lung cancer (Adjuvant Navelbine International Trialist Association [ANITA]): a randomised controlled trial. Lancet Oncol 2006;7:719-27.

4. Albain KS, Swann RS, Rusch VW, et al. Radiotherapy plus chemotherapy with or without surgical resection for stage III non-small-cell lung cancer: a phase III randomised controlled trial. Lancet 2009;374:379-86.

5. Antonia SJ, Villegas A, Daniel D, et al. Durvalumab after chemoradiotherapy in stage III non-small-cell Lung Cancer. N Engl J Med 2017;377:1919-29.

6. National Cancer Institute SSB. Cancer Incidence Surveillance, Epidemiology, and End Results (SEER) Registries Research Data 2007. Available online: http:// seer.cancer.gov/data/metadata.html

7. Non-small Cell Lung Cancer Collaborative Group. Chemotherapy in non-small cell lung cancer: a metaanalysis using updated data on individual patients from 52 randomised clinical trials. BMJ 1995;311:899-909.

8. Thomas A, Rajan A, Giaccone G. Tyrosine kinase inhibitors in lung cancer. Hematol Oncol Clin North Am 2012;26:589-605.

9. Maemondo M, Inoue A, Kobayashi K, et al. Gefitinib or chemotherapy for non-small-cell lung cancer with mutated EGFR. N Engl J Med 2010;362:2380-8.

10. Noonan SA, Camidge DR. PROFILE 1014: lessons for the new era of lung cancer clinical research. Transl Lung Cancer Res 2015;4:642-8.

11. Reck M, Rodríguez-Abreu D, Robinson AG, et al. Pembrolizumab versus Chemotherapy for PD-L1Positive Non-Small-Cell Lung Cancer. N Engl J Med 2016;375:1823-33.

12. Brahmer J, Reckamp KL, Baas P, et al. Nivolumab versus Docetaxel in Advanced Squamous-Cell Non-Small-Cell Lung Cancer. N Engl J Med 2015;373:123-35.

13. Borghaei H, Paz-Ares L, Horn L, et al. Nivolumab versus Docetaxel in Advanced Nonsquamous Non-Small-Cell Lung Cancer. N Engl J Med 2015;373:1627-39.

14. Fehrenbacher L, Spira A, Ballinger M, et al. Atezolizumab versus docetaxel for patients with previously treated non-small-cell lung cancer (POPLAR): A multicentre, open-label, phase 2 randomised controlled trial. Lancet 2016;387:1837-46.

15. Riedel S. Edward Jenner and the history of smallpox and vaccination. Proc (Bayl Univ Med Cent) 2005;18:21-5.

16. Coley WB. Contribution to the knowledge of sarcoma. Ann Surg 1891;14:199-220.

17. Nicol AF, De Andrade CV, Russomano FB, et al. HPV vaccines: Their pathology-based discovery, benefits, and adverse effects. Ann Diagn Pathol 2015;19:418-22.

18. Gonzalez G, Crombet T, Torres F, et al. Epidermal growth factor-based cancer vaccine for non-small-cell lung cancer therapy. Ann Oncol 2003;14:461-6.

19. Finn OJ. Cancer immunology molecular origins of cancer. N Engl J Med 2008;358:2704-15.

20. Decoster L, Wauters I, Vansteenkiste JF. Vaccination therapy for non-small-cell lung cancer: Review of agents in phase III development. Ann Oncol 2012;23:1387-93.

21. Kelly RJ, Giaccone G. Lung cancer vaccines. Cancer J 2011;17:302-8.

22. Finn OJ. Cancer vaccines: between the idea and the reality. Nat Rev Immunol 2003;3:630-41.

23. Freeman-Keller M, Goldman J, Gray J. Vaccine immunotherapy in lung cancer: Clinical experience and future directions. Pharmacol Ther 2015;153:1-9.

24. Ramlogan-Steel CA, Steel JC, Morris JC. Lung cancer vaccines: current status and future prospects. Transl Lung Cancer Res 2014;3:46-52.

25. Hirschowitz EA, Hiestand DM, Yannelli JR. Vaccines for lung cancer. J Thorac Oncol 2006;1:93-104.

26. Fredman P, Hedberg K, Brezicka T. Gangliosides as therapeutic targets for cancer. BioDrugs 2003;17:155-67.

27. Patra SK. Dissecting lipid raft facilitated cell signaling pathways in cancer. Biochim Biophys Acta 2008;1785:182-206.

28. Livingston P. Ganglioside vaccines with emphasis on GM2. Semin Oncol 1998;25:636-45.

29. Scanlan MJ, Gure AO, Jungbluth AA, et al. Cancer/ testis antigens: an expanding family of targets for cancer immunotherapy. Immunol Rev 2002;188:22-32.

30. Srivatsan S. Allogeneic tumor cell vaccines: the promise and limitations in clinical trials. Hum Vaccin Immunother 2014;10:52-63.

31. Suzuki H, Owada Y, Watanabe Y, et al. Recent advances in immunotherapy for non-small-cell lung cancer. Hum Vaccin Immunother 2014;10:352-7.

32. Miyazono K. Positive and negative regulation of TGF- 
beta signaling. J Cell Sci 2000;113:1101-9.

33. Nemunaitis J, Dillman RO, Schwarzenberger PO, et al. Phase II study of belagenpumatucel-L, a transforming growth factor beta-2 antisense gene-modified allogeneic tumor cell vaccine in non-small-cell lung cancer. J Clin Oncol 2006;24:4721-30.

34. Nemunaitis J, Nemunaitis M, Senzer N, et al. Phase II trial of Belagenpumatucel-L, a TGF-beta2 antisense gene modified allogeneic tumor vaccine in advanced non small cell lung cancer (NSCLC) patients. Cancer Gene Ther 2009;16:620-4.

35. Giaccone G, Bazhenova LA, Nemunaitis J, et al. A phase III study of belagenpumatucel-L, an allogeneic tumour cell vaccine, as maintenance therapy for non-small cell lung cancer. Eur J Cancer 2015;51:2321-9.

36. Melisi D, Garcia-Carbonero R, Macarulla T, et al. A phase II, double-blind study of galunisertib+gemcitabine (GG) vs gemcitabine+placebo (GP) in patients (pts) with unresectable pancreatic cancer (PC). J Clin Oncol 2016;34:abstr 4019.

37. Dessureault S, Noyes D, Lee D, et al. A phase-I trial using a universal GM-CSF-producing and CD40L-expressing bystander cell line (GM.CD40L) in the formulation of autologous tumor cell-based vaccines for cancer patients with stage IV disease. Ann Surg Oncol 2007;14:869-84.

38. Creelan BC, Antonia S, Noyes D, et al. Phase II trial of a GM-CSF-producing and CD40L-expressing bystander cell line combined with an allogeneic tumor cell-based vaccine for refractory lung adenocarcinoma. J Immunother 2013;36:442-50.

39. Gray JE, Chiappori A, Williams CC, et al. Phase I/ II randomized trial of GM.CD40L vaccine plus/minus CCL21 in advanced lung adenocarcinoma: Final results. J Clin Oncol 2016;34:9037.

40. Mendelsohn J, Baselga J. Epidermal growth factor receptor targeting in cancer. Semin Oncol 2006;33:369-85.

41. Luo SY, Lam DC. Oncogenic driver mutations in lung cancer. Transl Respir Med 2013;1:6.

42. Rotow J, Bivona TG. Understanding and targeting resistance mechanisms in NSCLC. Nat Rev Cancer 2017;17:637-58.

43. Saavedra D, Crombet T. CIMAvax-EGF: A new therapeutic vaccine for advanced non-small cell lung cancer patients. Front Immunol 2017;8:269.

44. Neninger Vinageras E, de la Torre A, Osorio Rodríguez M, et al. Phase II randomized controlled trial of an epidermal growth factor vaccine in advanced non-small-cell lung cancer. J Clin Oncol 2008;26:1452-8.
45. Rodriguez PC, Popa X, Martinez O, et al. A phase III clinical trial of the epidermal growth factor vaccine CIMAvax-EGF as switch maintenance therapy in advanced non-small-cell lung cancer patients. Clin Cancer Res 2016;22:3782-90.

46. Gjerstorff MF, Andersen MH, Ditzel HJ. Oncogenic cancer/testis antigens: prime candidates for immunotherapy. Oncotarget 2015;6:15772-87.

47. Grah JJ, Katalinic D, Juretic A, Santek F, Samarzija M. Clinical significance of immunohistochemical expression of cancer/testis tumor-associated antigens (MAGE-A1, MAGE-A3/4, NY-ESO-1) in patients with non-small cell lung cancer. Tumori 2014;100:60-8.

48. Vansteenkiste JF, Cho BC, Vanakesa T, et al. Efficacy of the MAGE-A3 cancer immunotherapeutic as adjuvant therapy in patients with resected MAGE-A3-positive nonsmall-cell lung cancer (MAGRIT): a randomised, doubleblind, placebo-controlled, phase 3 trial. Lancet Oncol 2016;17:822-35.

49. Gnjatic S, Nishikawa H, Jungbluth AA, et al. NY-ESO-1: Review of an immunogenic tumor antigen. Adv Cancer Res 2006;95:1-30.

50. Gure AO, Chua R, Williamson B, et al. Cancer-testis genes are coordinately expressed and are markers of poor outcome in non-small cell lung cancer. Clin Cancer Res 2005;11:8055-62.

51. John T, Starmans MH, Chen YT, et al. The Role of Cancer-Testis Antigens as Predictive and Prognostic Markers in Non-Small Cell Lung Cancer. PLoS One 2013;8:e67876.

52. Murphy R, Green S, Ritter G, et al. Recombinant NYESO-1 cancer antigen: Production and purification under cGMP conditions. Prep Biochem Biotechnol 2005;35:119-34.

53. Behzad H, Huckriede AL, Haynes L, et al. GLA-SE, a synthetic toll-like receptor 4 agonist, enhances T-cell responses to influenza vaccine in older adults. J Infect Dis 2012;205:466-73.

54. Neninger E, Diaz RM, de la Torre A, et al. Active immunotherapy with $1 \mathrm{E} 10$ anti-idiotype vaccine in patients with small cell lung cancer: report of a phase I trial. Cancer Biol Ther 2007;6:145-50.

55. Alfonso S, Diaz RM, De La Torre A, et al. 1E10 anti-idiotype vaccine in non-small cell lung cancer: Experience in stage IIIb/IV patients. Cancer Biol Ther 2007;6:1847-52.

56. Alfonso S, Valdés-Zayas A, Santiesteban ER, et al. A randomized, multicenter, placebo-controlled clinical trial 
of racotumomab-alum vaccine as switch maintenance therapy in advanced non-small cell lung cancer patients. Clin Cancer Res 2014;20:3660-71.

57. Ho SB, Niehans GA, Lyftogt C, et al. Heterogeneity of mucin gene expression in normal and neoplastic tissues. Cancer Res 1993;53:641-51.

58. Palmer M, Parker J, Modi S, et al. Phase I study of the BLP25 (MUC1 peptide) liposomal vaccine for active specific immunotherapy in stage IIIB/IV non-small-cell lung cancer. Clin Lung Cancer 2001;3:49-57.

59. Butts C, Murray N, Maksymiuk A, et al. Randomized phase IIB trial of BLP25 liposome vaccine in stage IIIB and IV non-small-cell lung cancer. J Clin Oncol 2005;23:6674-81.

60. Butts C, Maksymiuk A, Goss G, et al. Updated survival analysis in patients with stage IIIB or IV non-small-cell lung cancer receiving BLP25 liposome vaccine (L-BLP25): Phase IIB randomized, multicenter, open-label trial. J Cancer Res Clin Oncol 2011;137:1337-42.

61. Butts C, Socinski MA, Mitchell PL, et al. Tecemotide (L-BLP25) versus placebo after chemoradiotherapy for stage III non-small-cell lung cancer (START): A randomised, double-blind, phase 3 trial. Lancet Oncol 2014;15:59-68.

62. Ramalingam SS, Mitchell P, Vansteenkiste JF, et al. START2: Tecemotide in unresectable stage III NSCLC after first-line concurrent chemoradiotherapy. J Clin Oncol 2014;32:TPS7608.

63. Wu YL, Park K, Soo RA, et al. INSPIRE: A phase III study of the BLP25 liposome vaccine (L-BLP25) in Asian patients with unresectable stage III non-small cell lung cancer. BMC Cancer 2011;11:430.

64. Ohyanagi F, Horai T, Sekine I, et al. L-BLP25 for unresectable stage III non-small cell lung cancer after primary chemoradiotherapy in Japanese patients: Preliminary safety results from a phase I/II study. J Clin Oncol 2010;28:e13153.

65. Stockwin LH, McGonagle D, Martin IG, et al. Dendritic cells: immunological sentinels with a central role in health and disease. Immunol Cell Biol 2000;78:91-102.

Cite this article as: Oliveres $\mathrm{H}$, Caglevic C, Passiglia F, Taverna S, Smits E, Rolfo C. Vaccine and immune cell therapy in non-small cell lung cancer. J Thorac Dis 2018;10(Suppl 13):S1602-S1614. doi: 10.21037/jtd.2018.05.134
66. Banchereau J, Palucka AK. Dendritic cells as therapeutic vaccines against cancer. Nat Rev Immunol 2005;5:296-306.

67. Ponomarenko DM, Klimova ID, Chapygina YA, et al. Safety and efficacy of p62 DNA vaccine ELENAGEN in a first-in-human trial in patients with advanced solid tumors. Oncotarget 2017;8:53730-9.

68. Limacher JM, Quoix E. TG4010: A therapeutic vaccine against MUC1 expressing tumors. Oncoimmunology 2012;1:791-2.

69. Rochlitz C, Figlin R, Squiban P, et al. Phase I immunotherapy with a modified vaccinia virus (MVA) expressing human MUC1 as antigen-specific immunotherapy in patients with MUC1-positive advanced cancer. J Gene Med 2003;5:690-9.

70. Ramlau R, Quoix E, Rolski J, et al. A phase II study of Tg4010 (Mva-Muc1-Il2) in association with chemotherapy in patients with stage III/IV Non-small cell lung cancer. J Thorac Oncol 2008;3:735-44.

71. Quoix E, Ramlau R, Westeel V, et al. Therapeutic vaccination with TG4010 and first-line chemotherapy in advanced non-small-cell lung cancer: A controlled phase 2B trial. Lancet Oncol 2011;12:1125-33.

72. Quoix E, Lena H, Losonczy G, et al. TG4010 immunotherapy and first-line chemotherapy for advanced non-small-cell lung cancer (TIME): Results from the phase $2 \mathrm{~b}$ part of a randomised, double-blind, placebocontrolled, phase 2b/3 trial. Lancet Oncol 2016;17:212-23.

73. Robbins PF, Morgan RA, Feldman SA, et al. Tumor regression in patients with metastatic synovial cell sarcoma and melanoma using genetically engineered lymphocytes reactive with NY-ESO-1. J Clin Oncol 2011;29:917-24.

74. Rapoport AP, Stadtmauer EA, Binder-Scholl GK, et al. NY-ESO-1-specific TCR-engineered T cells mediate sustained antigen-specific antitumor effects in myeloma. Nat Med 2015;21:914-21.

75. Li N, Liu S, Sun M, et al. Chimeric antigen receptormodified T cells redirected to EphA2 for the immunotherapy of non-small cell lung cancer. Transl Oncol 2018;11:11-7. 